\title{
Focused Ion Beam Induced X-Ray Analysis
}

\author{
Lucille A. Giannuzzi
}

FEI Company, 5350 NE Dawson Creek Drive, Hillsboro, OR 97124 USA

Characteristic x-rays may be emitted as the result of ion-solid interactions and is the mechanism for particle induced $\mathrm{x}$-ray analysis (PIXE). Conventional PIXE techniques use light ions or protons accelerated at energies in the $\mathrm{MeV}$ range [1]. However, $\mathrm{x}$-ray emission has also been documented for heavy ions accelerated to a few $\mathrm{keV}$ [2]. The advantage to using heavy ions is that the collision cascade occurs close to the surface, and therefore, direct surface analysis and/or accurate depth profiling could be accomplished. In addition, since no continuum or Bremmstralung is generated by ion-solid interactions, ion beam induced x-ray analysis may be orders of magnitude more sensitive than electron beam induced $x$-ray analysis [2]. Previous results have also indicated that there is a characteristic cut-off energy which is ion and target dependent, above which no x-rays are emitted [2]. The collection of soft $\mathrm{x}$-rays $(<\sim 2 \mathrm{keV})$ are most sensitive to PIXE, and thus, appropriate for light element analysis. Heavy ions (e.g., $\mathrm{Ga}^{+}$) in the $\mathrm{keV}$ energy range are readily available with commercial focused ion beam (FIB) instruments. Thus, the efficacy of FIB induced x-ray analysis (FIBIX) as an analytical technique is discussed below.

An FEI Nova NanoLab 600 DualBeam (FIB + scanning electron microscope (SEM)) equipped with an EDAX x-ray detector was used to collect x-ray energy dispersive spectra (XEDS) using $30 \mathrm{keV}$ $\mathrm{Ga}^{+}$ions at a beam current of $20 \mathrm{nA}$. Carbon coated NIST standard reference materials 1872 containing varying amounts of $\mathrm{Ge}, \mathrm{Pb}$, and $\mathrm{O}$, were analyzed. The reference standards were mounted on a $45^{\circ}$ pre-tilted holder and rotated such that their plane normal was equidistant between the FIB column and the XEDS detector.

FIG. 1 shows the superposition of XEDS profiles acquired in $100 \mathrm{~s}$ from sample K968 using either the FIB as the primary source (red) or the SEM as the primary source (blue). Note that the FIBIX spectrum drops to below 4 counts at $\sim 4 \mathrm{keV}$ which is much less than the incident $30 \mathrm{keV} \mathrm{Ga}^{+}$ions as predicted by theory. The primary constituents of $\mathrm{O}, \mathrm{Ge}$, and $\mathrm{Pb}$ are evident in both the FIBIX and SEM spectra. A continuously decreasing Bremmstralung is noted in the FIBIX spectrum, and the large consistent Bremmstralung is noted in the SEM spectrum, particularly at the higher energies [3].

\section{References}

[1] J.W. Martin, The Local Chemical Analysis of Materials, Elsevier, Amsterdam, (2003).

[2] J.A. Cairns, Surface Science, 34 (1973), 638.

[3] Special thanks to Mike Bernas, Trisha Rice, and Richard Young for helpful discussions. 


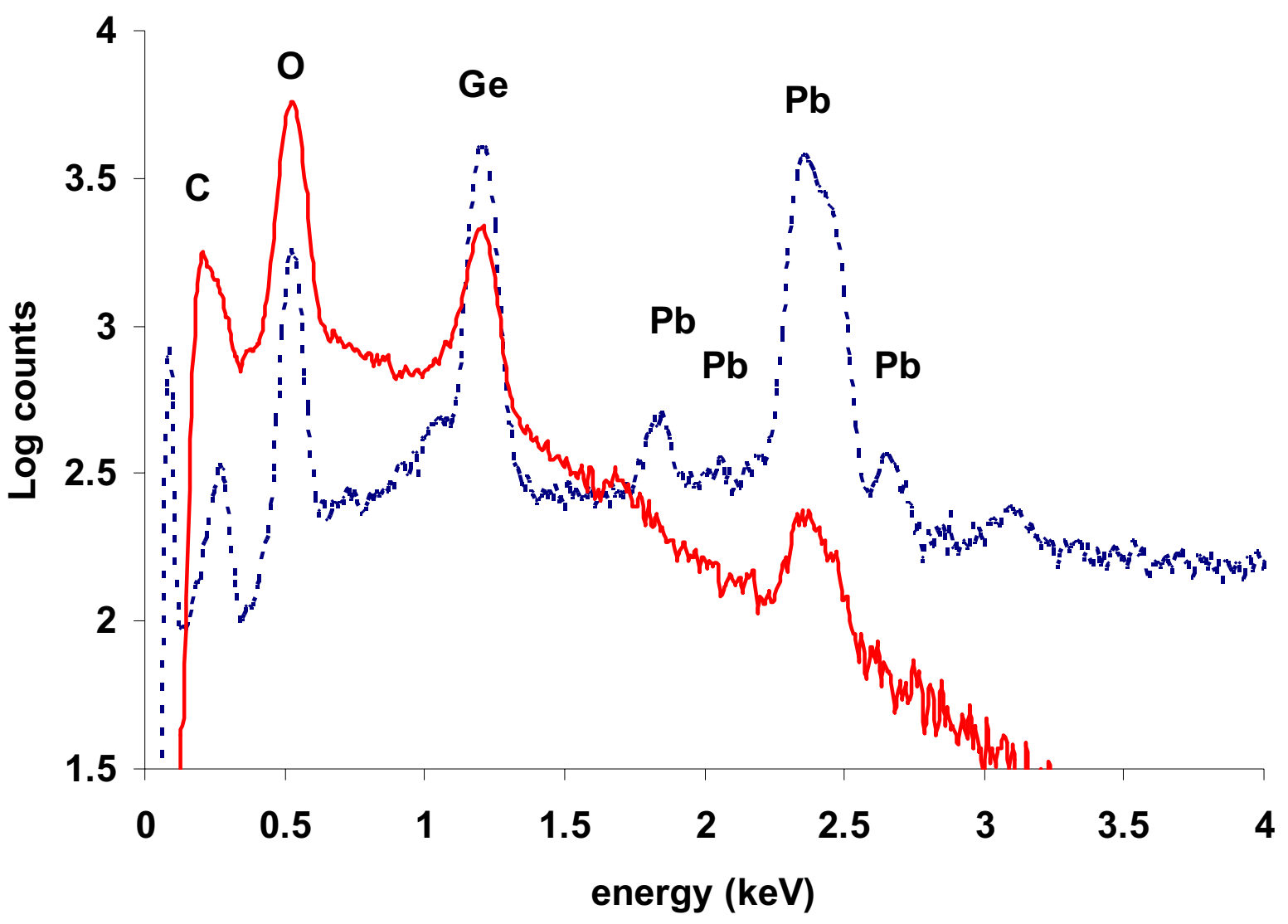

FIG 1. XEDS spectra obtained in $100 \mathrm{~s}$ using a FIB (red) or SEM (blue) as the primary source. 